http://revistaurbanismo.uchile.cl

\title{
San Fernando en el siglo XX: de la ciudad concentrada al mosaico urbano
} The city of San Fernando in the $20^{\text {th }}$ Century: from a compact city to an urban mosaic

\section{Autores}

Leonel Pérez Bustamante y Maritza Saavedra Meléndez

\section{Filiación}

Departamento de Urbanismo y Centro Eula de la Universidad de Concepción.

\section{Resumen}

La escasez de material acerca de la evolución reciente de la forma urbana de la ciudad de San Fernando, Región del Libertador Bernardo O'Higgins, ha sido la principal motivación para llevar a cabo este trabajo de investigación, que ha buscado traer a la memoria sus principales momentos urbanos.

Se inicia cuando los efectos de calidad y cantidad de los procesos de urbanización (González y Romero, 2005) (González et al, 2004) y efectos de la agroindustrialización se hacen evidentes en su estructura urbana. La ciudad se transforma paulatina y sostenidamente, transformando también sus propios espacios urbanos.

\section{Palabras clave}

Formas de crecimiento, fragmentación urbana, forma urbana

\section{Abstract}

This paper presents the evolution of the urban shape of San Fernando, modified by the new proccess of housing and agriculture.

\section{Keywords}

Urban growth, Urban Sprawl, Urban Shape.

\section{Sumario}

0.- Introducción

1.- La ciudad en Progreso: 1942-1972

2.- La ciudad de Contornos: 1973-1989

3.- La ciudad en Expansión: 1990-2008

4.- Conclusión

5.- Bibliografía 


\section{Introducción.}

Fernández (2001:17) reconoce que una ciudad es obra de todos los días, pero simultáneamente afirma que es siempre un hecho histórico, con mayor o menor profundidad, distancia temporal o intensidad, pero siempre es historia (Korn, 1963). Basado en ello, la investigación acerca de la ciudad se desarrolla en orden cronológico desde el año 1942 hasta el año 2008.

También la ciudad es vista como una espacialización de la historia (Fernández, 2001:18). El método utilizado ha partido de la consulta a los expedientes de construcción existentes en la I. Municipalidad de San Fernando; desarrollando a partir de este material el estudio de momentos y secuencias de construcción material de la ciudad en los que se hace presente su historia. Los resultados del estudio se presentan en tres apartados que analizan el crecimiento y la evolución de la forma de la ciudad en distintos momentos históricos, identificables tanto en la propia ciudad como en un contexto más amplio: 1) la ciudad en Progreso (1942-1972), b) ciudad de Contornos (1973-1989), y, c) la ciudad en Expansión: 1990-2008.

Analizar la ciudad de San Fernando en su pasado reciente servirá no solo para un conocimiento de su situación actual; sino que además permite aportar a la comprensión de otras ciudades intermedias de valle central (Olave, 2005), entendido como un viaje de la historia de la ciudad chilena en el último siglo ${ }^{1}$.

\section{La ciudad en Progreso: 1942-1972.}

A comienzos de los años cuarenta, San Fernando tenía como hito norte la casa patronal del fundo Nincunlauta y sus terrenos, al nororiente la ciudad, los cuales se desarrollan tangencialmente a la Avda. Manso de Velasco distinguiéndose el Estadio Municipal, al norponiente el Cementerio y la calle Juan Jiménez, hacia el Sur la calle Los Palacios y hacia el suroriente la ciudad traspasa la línea férrea con la población San Martín, a su costado Molino el Cisne y siguiendo por la calle Avda. José Miguel Carrera se ubica el regimiento de Colchagua, ubicado estratégicamente en el límite de la ciudad.

\footnotetext{
${ }^{1}$ Para profundizar sobre el origen y desarrollo previo de algunas de estas ciudades (Quillota, los Ángeles, San Felipe, Cauquenes, Talca, San Fernando, Melipilla, Rancagua, Curicó, Copiapó) resulta imprescindible ver el estudio de LORENZO, 1995. 
San Fernando en el siglo XX: de la ciudad concentrada al mosaico urbano
ISSN $0717-5051$

http://revistaurbanismo.uchile.cl
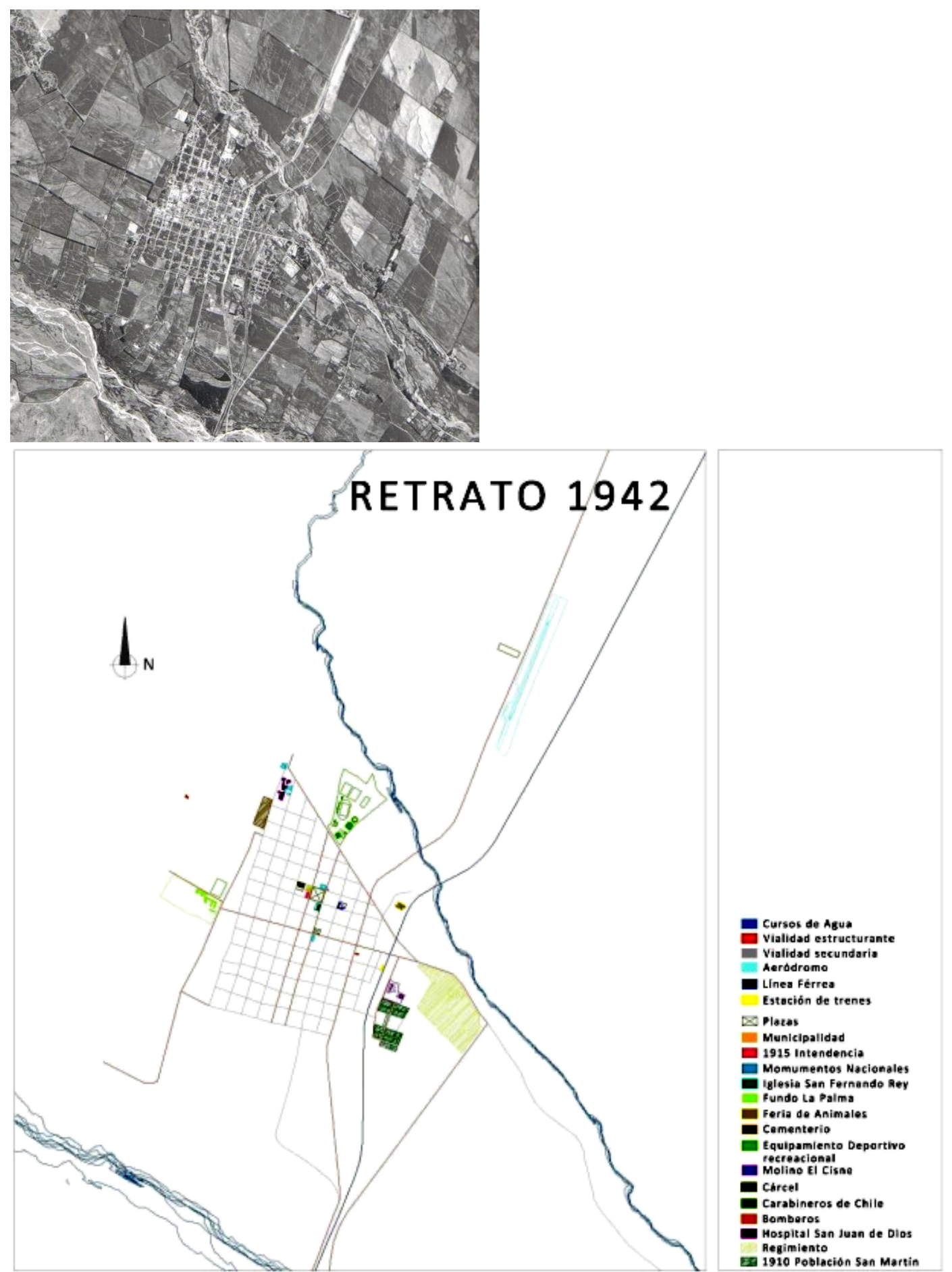

Fig. 1. Fotografía Aérea 22 de Enero de 1955. Fuente: Instituto Geográfico Militar (IGM)

Fig. 2. Retrato de la ciudad en 1942. Fuente: elaboración M. Saavedra. 
Esta década está marcada por un avance en materias educacionales destacando la construcción de la Escuela de Hombres ubicada en calle Cardenal Caro a una cuadra al oriente de la Plaza de Armas en 1942. (Fig. 2).

Hasta mediados de la década, la ciudad mantenía su cuadrícula fundacional con algunos ensanches y proyecciones, se moderniza paulatinamente y en 1945 se construye la Longitudinal Norte permitiendo la conexión expedita de la ciudad hacia Santiago.

A principios de la década del 1950 el Estado forma la Corporación de la Vivienda (CORVI) y el Plan Nacional de la Vivienda, los que promueven la concentración urbana como respuesta a la mayor demanda de viviendas.

En 1950 se inicia la construcción de la Población Molino San Cristóbal, ubicada en la calle Arturo Prat adyacente al Molino el Cisne y la Compañía de Petróleos de Chile (ENAP) en la proyección de calle José Miguel Carrera (continuación de Manuel Rodríguez a partir de la línea férrea y en dirección suroriente), generando a la postre una proyección y un área de crecimiento urbano en dirección hacia la cordillera de Los Andes.

En 1952 se construye el Mercado Municipal situando sus fachadas hacia las calles Chacabuco y España.

La ciudad estaba en proceso de consolidación a nivel educacional aumentando la dotación de Escuelas y Liceos (Liceo de Niñas, Escuela Hogar Femenina, Escuelas Técnicas).

El centro urbano se densifica con la aparición de nuevos conjuntos residenciales como la Población Neandro Schilling y Población San Fernando (ambas de 1957) (Fig. 2).

En 1959 se promulga la Ley del Plan Habitacional (Hidalgo, 2005). En este periodo se impulsó la acción de empresas privadas de la construcción y se institucionalizó el ahorro para la vivienda, se establecieron tipologías de viviendas adecuadas a la capacidad de cada familia, se llamó a concurso para el diseño de viviendas sociales, y se intentaba establecer bases para la radicación y erradicación de los asentamientos espontáneos.

Por medio de la Operación Sitio se promueve la autoconstrucción, desde un sitio con urbanización mínima que buscaba ser completado en el tiempo (muchas de éstos quedaron inconclusas en urbanización y títulos de dominio).

A principios de 1960 se reconocía en la ciudad una zona de crecimiento al oriente de calle Manso de Velasco en los costados del Estadio Municipal, conformada por la Población 18 de Septiembre (1960 aprox.) y por la Villa Olímpica (1966). Además se observa una nueva zona al oriente de la calle José Miguel Carrera, formada por las 
San Fernando en el siglo XX: de la ciudad concentrada al mosaico urbano The city of San Fernando in the 20th Century: from a compact city to an urban mosaic

http://revistaurbanismo.uchile.cl

Poblaciones General Carrera y Santa Elena (ambas en 1960 aprox.). Esta nueva franja de crecimiento traslada los bordes de la ciudad hacia el estero Antivero.

En este mismo periodo se traza la Panamericana Sur, la cual es trazada perpendicular a la calle José Miguel Carrera conectando la ciudad hacia el Sur, conformando una importante intersección urbana y una zona excéntrica de crecimiento de la ciudad hacia el suroriente completada por la instalación en este punto de la empresa Chiprodal en 1963 (actualmente Nestlé) y sus etapas sucesivas, constituyéndose en una imagen industrial representativa de la ciudad de fuerte presencia hasta la actualidad.

En 1965 se creó el Ministerio de Vivienda y Urbanismo, el que tuvo como objetivo abordar las materias referidas al desarrollo urbano (Hidalgo, 2005). La posterior creación de la Oficina Nacional de Planificación (ODEPLAN), aportó con una nueva visión económica de la gestión urbana, sin embargo parcializada en las distintas materias (educación, salud, vivienda, etc.).

En el año 1969 se recepciona la Población San Fernando sector Nincunlauta, al norponiente de la casa patronal del mismo nombre; cuya conectividad está dada por la calle Feliciano Silva, ubicada a cuatro cuadras al poniente de Juan Jiménez. Este ensanche originó una tensión de desarrollo residencial, el cual vino a consolidarse con la construcción de la Población Centinela (1970) como una franja paralela adosada a Juan Jiménez y aproximándose a la Población San Fernando. Ese mismo año se consolida el crecimiento del la franja paralela a la calle José Miguel Carrera con la construcción de las poblaciones Colchagua (1966), Cooperativa Marista (1969), Villa Antivero (1971), Compañía Chilena de Tabacos y Regimiento de Colchagua (1969), emplazadas de poniente a oriente.

El comienzo de la década de 1970 y hasta 1973 está marcado por los movimientos y políticas sociales impulsadas por el gobierno de la época. En un principio, las administraciones públicas se resistieron a soluciones paliativas a estos problemas, pero finalmente plantearon Planes de Emergencia que consistían en construcción de viviendas y urbanización, pero sin reajustes sobre las deudas hipotecarias de los grupos más pobres.

El Estado tomó en sus manos la producción de viviendas, participó en el mercado del suelo urbano y en el proceso de la construcción promoviendo el uso de conceptos como prefabricación o sistemas industrializados. En este periodo el gobierno evita la intervención del mundo privado y particulares en estos ámbitos.

Se construyen viviendas para obreros y empleados en sectores cercanos a los lugares de trabajo.

En este periodo se observa una consolidación y densificación de la ciudad por medio de poblaciones construidas dentro del centro histórico de la ciudad y en la zona aledaña a la vía férrea. Aparecía la primera población de dimensiones importantes en el eje Los Palacios, límite sur del casco histórico de la ciudad: la Población Colchagua Sur (1972). 
La ciudad de San Fernando cambiaba sus estructuras coloniales basadas en el desplazamiento a caballo por estructuras modernas de trenes y automóviles.
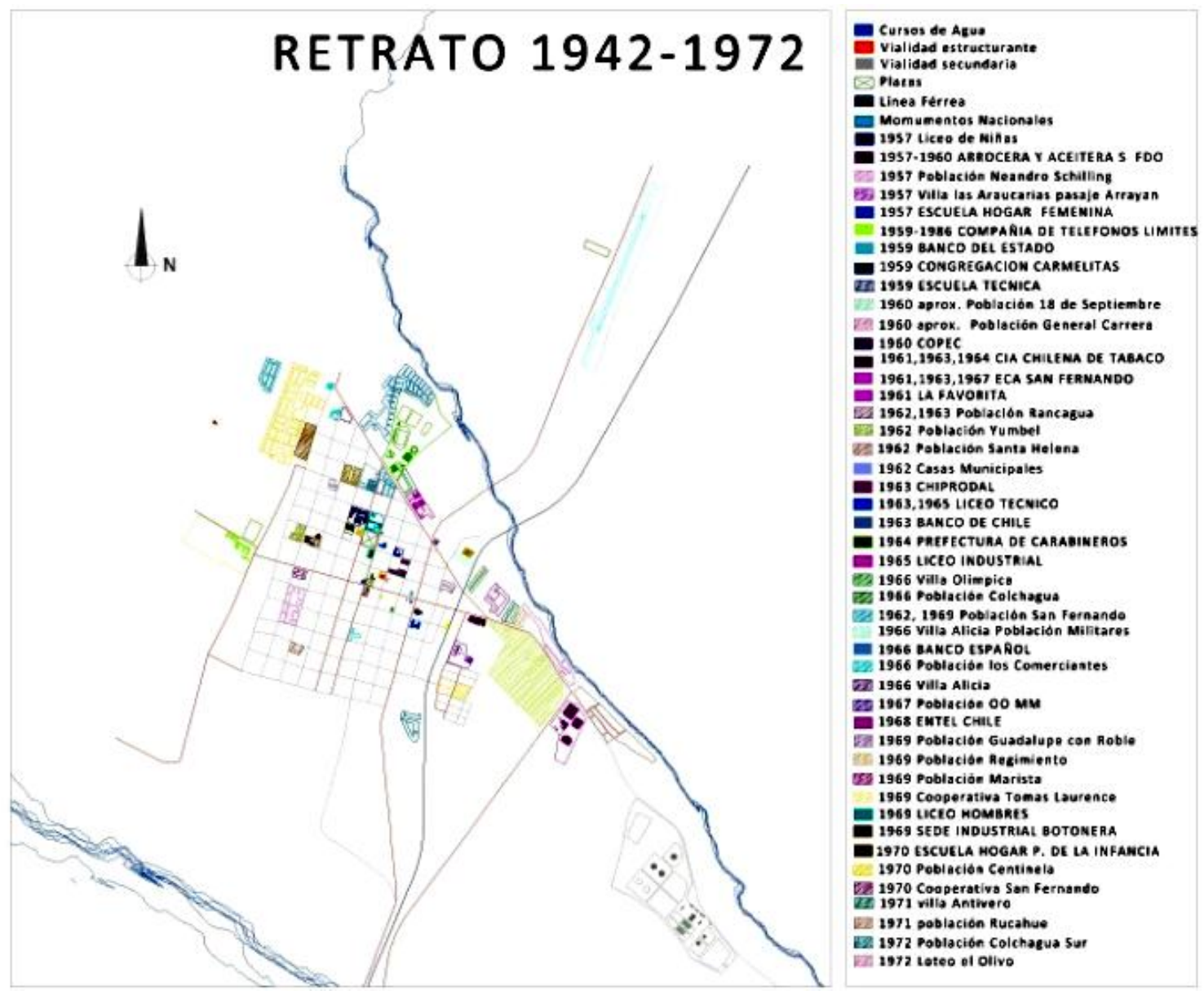

Fig. 3. Retrato de la ciudad período 1942-1972. Fuente: elaboración M. Saavedra.

La conectividad vial posibilitada por la longitudinal norte, vino a unir el país, utilizando la Avenida Bernardo O'Higgins como eje de traspaso Norte-Sur al interior de la ciudad, eje urbano que en el cruce con Avenida Manuel Rodríguez, consolidó como el centro comercial y de servicios.

Las migraciones y el crecimiento sostenido de la población, hacen que las ciudades cambien y experimenten la necesidad de cobijar a sus habitantes y sus requerimientos como educación, recreación, intercambio y vivienda principalmente.

San Fernando se consolidaba por medio de la construcción de colegios, poblaciones, servicios públicos y empresas principalmente Agroindustriales; pasa de ser ciudad 
básica a ciudad equipada, dejando atrás la preocupación acerca de la cantidad de población para pasar a un interés por la calidad de vida. (Fig. 3).

A finales de 1972 es posible reconocer tres áreas de crecimiento:

\section{- $\quad$ Área A. Norponiente Casa Patronal Nincunlauta.}

Esta área se constituye en primera instancia a partir de una unidad aislada: población San Fernando la cual provoca una tensión de crecimiento desplazando el borde de la ciudad hacia ésta por medio de la Población Centinela integrando a la trama el Cementerio de la ciudad, antes constituido como límite Norponiente.

\section{- Área B. Nororiente de Manso De Velasco en los costados del} Estadio Municipal.

Esta área se presenta como el remate de la circulación desde la Plaza de Armas hacia el Estadio Municipal (principales espacios públicos de la ciudad), por calle Carampangue, vía que alberga una concentración de colegios y liceos e institutos de la ciudad, densificándose de forma importante esta parte del centro histórico de la ciudad.

\section{- $\quad$ Área C. Nororiente de la calle José Miguel Carrera a los costados} de la Panamericana Sur.

Esta área se emplaza a modo de franja adosada a la vía de ingreso a la ciudad desde el sur a modo de borde hacia el Estero Antivero y adyacente a las nuevas zonas de crecimiento industrial y energético en dirección a la Cordillera, originando mixturas de usos de suelo y tramas residenciales diversas.

San Fernando comienza a desbordar sus límites por medio de unidades (residenciales e industriales) que se configuran con trazados irregulares, sin relación formal con la estructura original de cuadrícula; desprotegidas y expuestas a los vientos y crecidas estivales, dando nacimiento a una nueva estructura urbana de San Fernando: La ciudad histórica y su Borde estero. (Fig. 4. Zonas). 


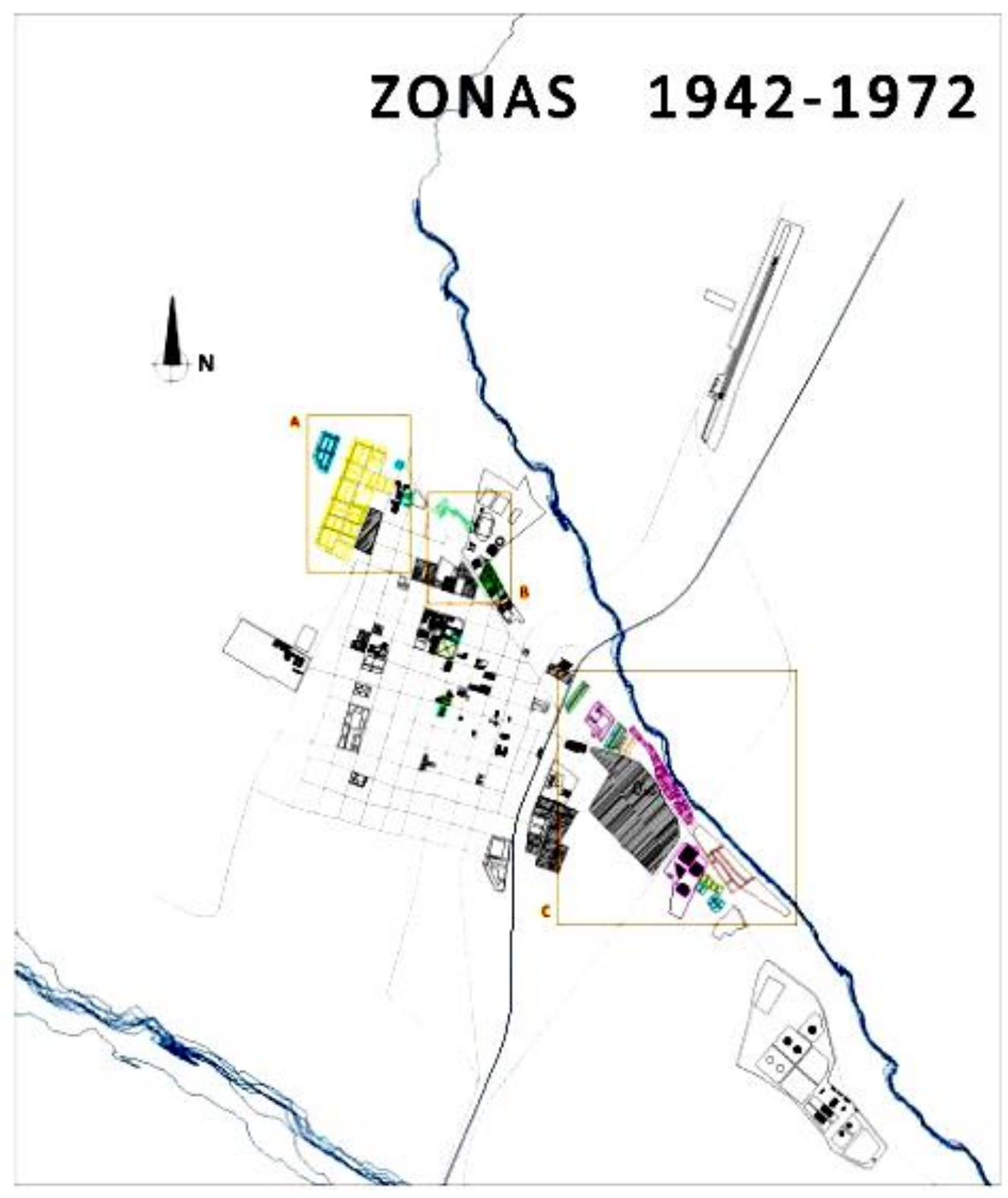

Fig. 4. Tres zonas o áreas de crecimiento 1942-1972. Fuente: elaboración M. Saavedra. 


\section{REVISTA DE}

$\bigcup_{\text {ISSN }} \operatorname{l717-5051} \mathrm{AN}$ ISMO

San Fernando en el siglo XX: de la ciudad concentrada al mosaico urbano

http://revistaurbanismo.uchile.cl

\section{La ciudad de Contornos: 1973-1989.}

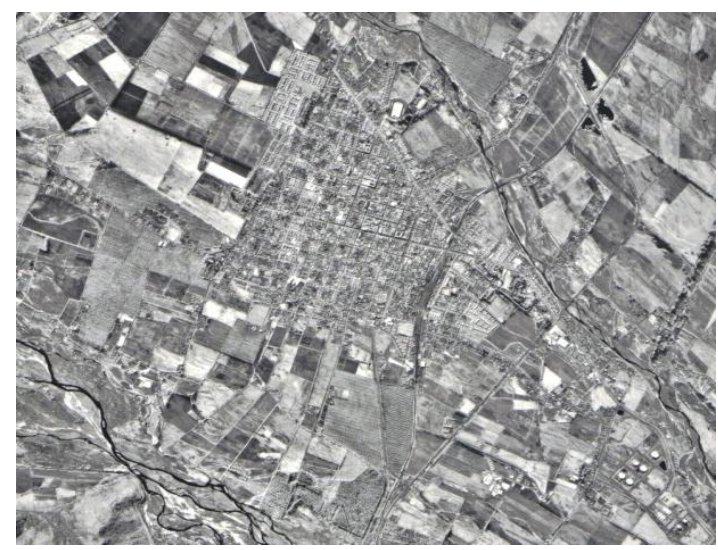

Fig. 5. Fotografía aérea, 1978. Fuente: Servicio Aerofotogramétrico (SAF) Fuerza Aérea de Chile.

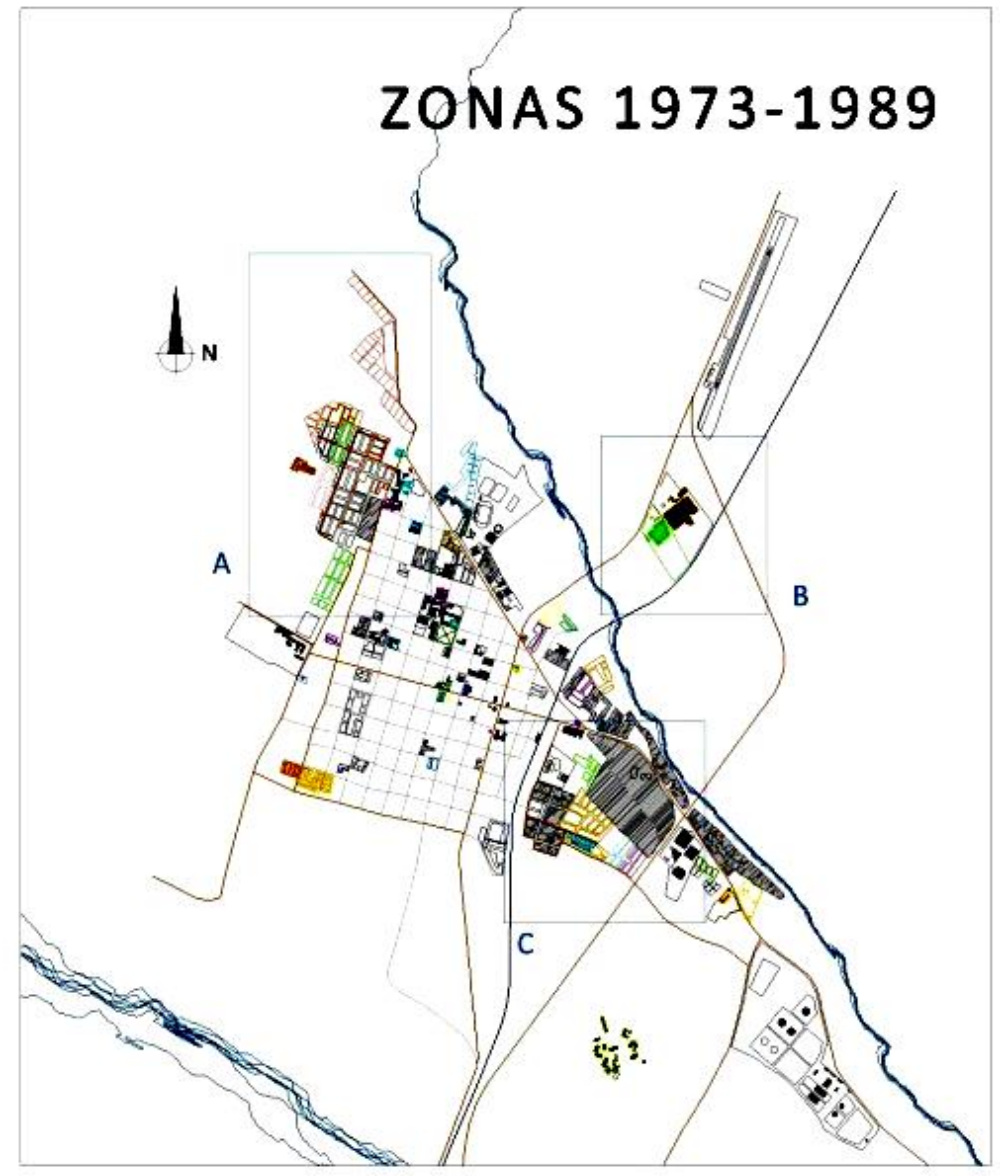

Fig. 6. Retrato de la ciudad período 1973-1989. Fuente: elaboración M. Saavedra. 
San Fernando en el siglo XX: de la ciudad concentrada al mosaico urbano The city of San Fernando in the 20th Century: from a compact city to an urban mosaic

http://revistaurbanismo.vchile.cl

El periodo definido por el gobierno militar (1973-1989) fue un momento de reformulación de la política de vivienda y urbanismo, dentro de los más relevantes destacan el Programa de vivienda Básica y la Liberación del Límite Urbano en Santiago.

En este periodo se estableció el modelo neoliberal pasando a ser los capitales privados los encargados del desarrollo y la planificación del país, instaurándose la subsidiariedad respecto de temas sociales.

En este etapa se observa el fortalecimiento de la nueva franja de crecimiento nororiente paralela al Estero Antivero por medio de las construcciones de la Villa Andrés Bello (1973), la extensión de la Población 18 de Septiembre (1975), la Villa Los Maitenes (1976) y la Villa Ensenada (1977). Se observa además una expansión urbana a continuación de la Población San Martín, al sur del Regimiento conformada por la Población Lautaro (1976-1977) lo que evidencia una ocupación paulatina hacia la carretera que une San Fernando con el sur de Chile.

En esta década se parcelan terrenos agrícolas en la zona norte de la ciudad, sector de Nincunlauta, integrándolos a la trama urbana y fortaleciendo el crecimiento adyacente al Estero Antivero.

En 1979 se promulga la Política Nacional de desarrollo urbano, la que liberalizó el mercado del suelo otorgándole la cualidad de bien no escaso, contemplando el crecimiento de las ciudades según la demanda y promoviendo la ocupación de nuevas áreas. Esta política por su énfasis en lo económico, olvida los temas sociales y los inconvenientes de la segregación.

En 1985 se observa la consolidación del crecimiento de la ciudad al Norponiente del Cementerio, alrededor del Estadio Municipal, a los costados poniente y sur del Regimiento de Colchagua.

En 1985 se pone en marcha una nueva Política de Desarrollo Urbano la cual establece que el suelo es un bien escaso, limitando la expansión del límite urbano. Se establece que es mejor densificar que crecer, sin embargo, el alto costo de los suelos urbanos centrales no permitió que prosperaran las ideas de renovación en las áreas deterioradas del casco histórico, produciéndose un crecimiento periférico sostenido, sectorizando la ciudad.

En 1985 se observa en la ciudad un incremento en la franja paralela al estero Antivero por medio de la expansión de la Población 18 de Septiembre (1979), hacia el Norponiente al costado del Cementerio aparece la Población 11 de Septiembre (1976), la Población Independencia (1983), Población Nueva Centinela (1983) y la Villa Conavicoop (1985).

En tanto, aparece la segunda población de grandes dimensiones adyacente a calle Los Palacios en los costados de la calle Negrete: la Población O'Higgins (1986-1987), que se convierte en catalizadora de una nueva zona de crecimiento de la ciudad hacia el sur. 
En 1989 la construcción de la Villa Real completa el espacio disponible desde la Población Lautaro hacia la carretera, consolidando la zona suroriente de la ciudad.

A finales de 1989 se distinguen tres áreas:

- Área A. Norponiente Población Centinela y camino Nincunlauta.

Esta área va creciendo desde los límites de la ciudad hacia su exterior, por medio de conjuntos habitacionales unitarios y de tramas irregulares disociados entre sí. Éstas en su conjunto conforman un tejido residencial de pequeñas ciudadelas.

\section{- Área B. Parque industrial}

Zona definida por la vía férrea y la carretera Panamericana desde el Estero Antivero en dirección al Norte. Se constituye como una excelente plataforma de negocios dada su conectividad y disponibilidad de espacio para grandes infraestructuras de producción y almacenaje de productos agrícolas. Posee un orden longitudinal de grandes predios con volúmenes aislados, además, la magnitud de sus terrenos permite el diseño de barreras verdes compuestas por bellos jardines.

- Área C. Cuadrante: Línea Férrea / Camino Real/ Panamericana Sur /el Regimiento de Colchagua.

Esta zona de crecimiento de la ciudad se desarrolla circunscrita por las vías, completando el espacio baldío por medio de franjas paralelas a la carretera desde la Población San Martín.

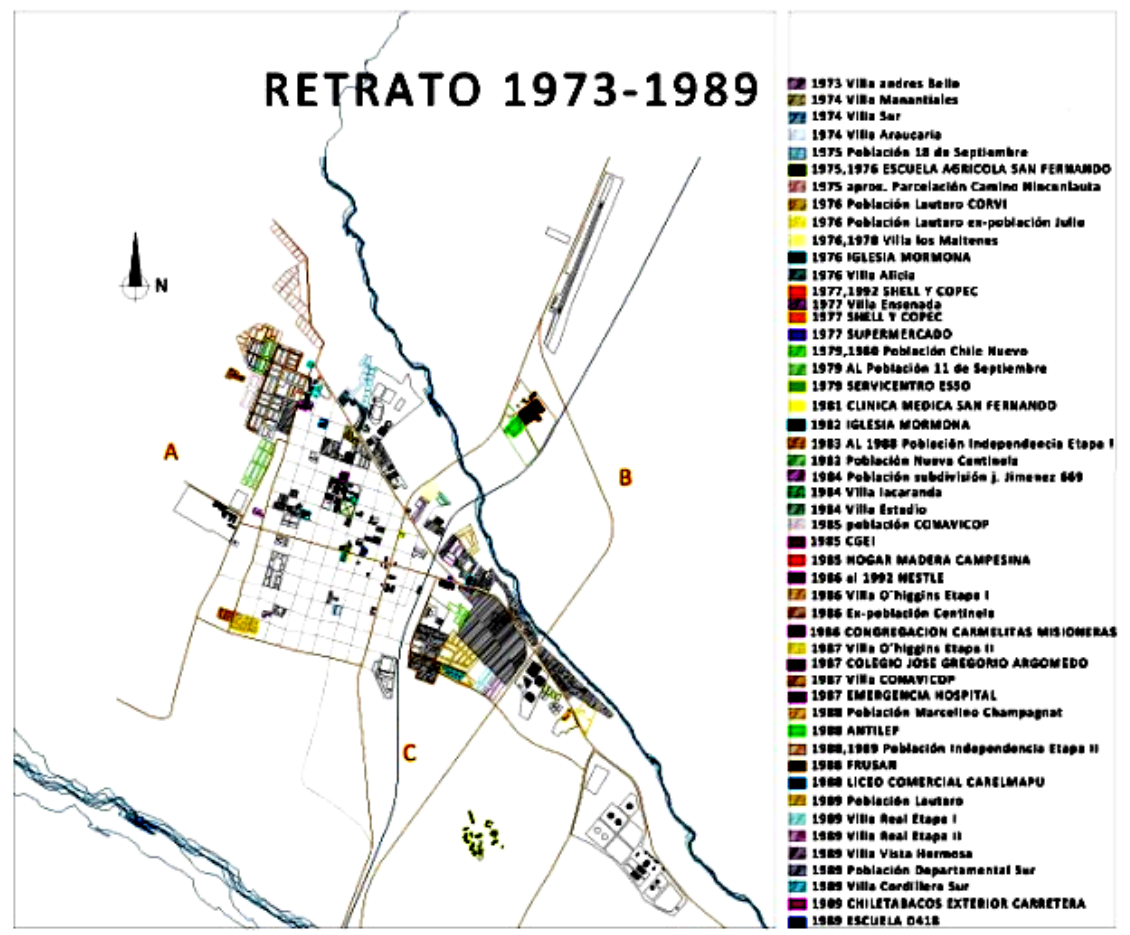

Fig. 7. Tres zonas o áreas de crecimiento 1973-1989. Fuente: elaboración M. Saavedra. 
San Fernando crece en un orden longitudinal a sus cursos de agua: estero Antivero y río Tinguiririca, sin embargo manifiesta una tendencia de crecimiento transversal, determinada por la influencia de la Panamericana Sur, por la vía férrea y por su Avda. Bernardo O'Higgins: eje de traspaso principal de la ciudad, paso desde la costa hacia Santiago y detención obligada de buses interurbanos.

Simultáneamente, la ciudad se constituye como un centro de servicios y abastecimiento de los poblados turísticos pre-cordilleranos provocando a su vez la intersección con el eje principal del país. Esta intersección promueve la actividad económica de la comuna.

\section{La ciudad en Expansión: 1990-2008.}

En este periodo se aumenta el gasto público en vivienda, se amplía el horizonte respecto de la autoconstrucción y se promueve la renovación urbana.

En esta etapa, la ciudad consolida el crecimiento hacia el sector norte a ambos lados del Camino Nincunlauta; prosiguiendo la ocupación de borde del estero Antivero, por medio de la Villa España (1991-1992) y la extensión de la Población 18 de Septiembre (1991).

El año 1991 la ciudad recibe en su trama el Terminal de Buses ubicado en calle Rancagua con Manso de Velasco, equipamiento inexistente previo a esta fecha. En este mismo sector surgen nuevos conjuntos residenciales que van rellenando los espacios libres de la franja de borde, tales como Villa San Marcelino (1992) y Villa Don Giusseppe (1993).

En 1995 la ciudad traspasa su límite sur definido por el callejón Los Palacios, con la construcción del Instituto San Fernando de educación, preescolar, básica y media; estableciendo un nuevo polo de desarrollo hacia el río Tinguiririca, apoyado por la instalación de una agroindustria de carácter inofensivo (Vitafood) (1990), y del Parque Cementerio Unidos (1992).

El año 1995 se construye el condominio Club de Polo, el cual se ordena en torno a su propia explanada privada, constituyéndose en un conjunto de características particulares en su entorno y en la ciudad. Se establece como un nuevo polo de crecimiento residencial en la proyección de la calle Valdivia al sur.

Al nororiente de la ciudad se proyectan dos nuevas vías paralelas y con ello una zona de crecimiento en donde se construyen conjuntos "SERVIU" en numerosas etapas:

- En calle El Roble se emplazan las poblaciones El Roble (1995), la Huerta (1995), Gabriela Mistral (1996), Villa Parque Lauca (2000) y Villa Origen (2007)

- En calle El Álamo se construyen Villa la Palma (1992), Doña Ester (2000), Villa el Álamo (1997-2003), Villa Doña Georgina (2002-2003).

Estas vías se proyectan perpendicularmente a la calle Juan Jiménez estableciendo una franja de crecimiento paralela. 
Durante la primera década del siglo XXI la ciudad ha traspasado el límite sur (callejón Los Palacios), estableciendo una zona de alta densidad en la proyección de Juan Jiménez por medio de Villa Don Darío (2000-2008), colindante a población Los Palacios y Villa San Marcos (2003- 2007).

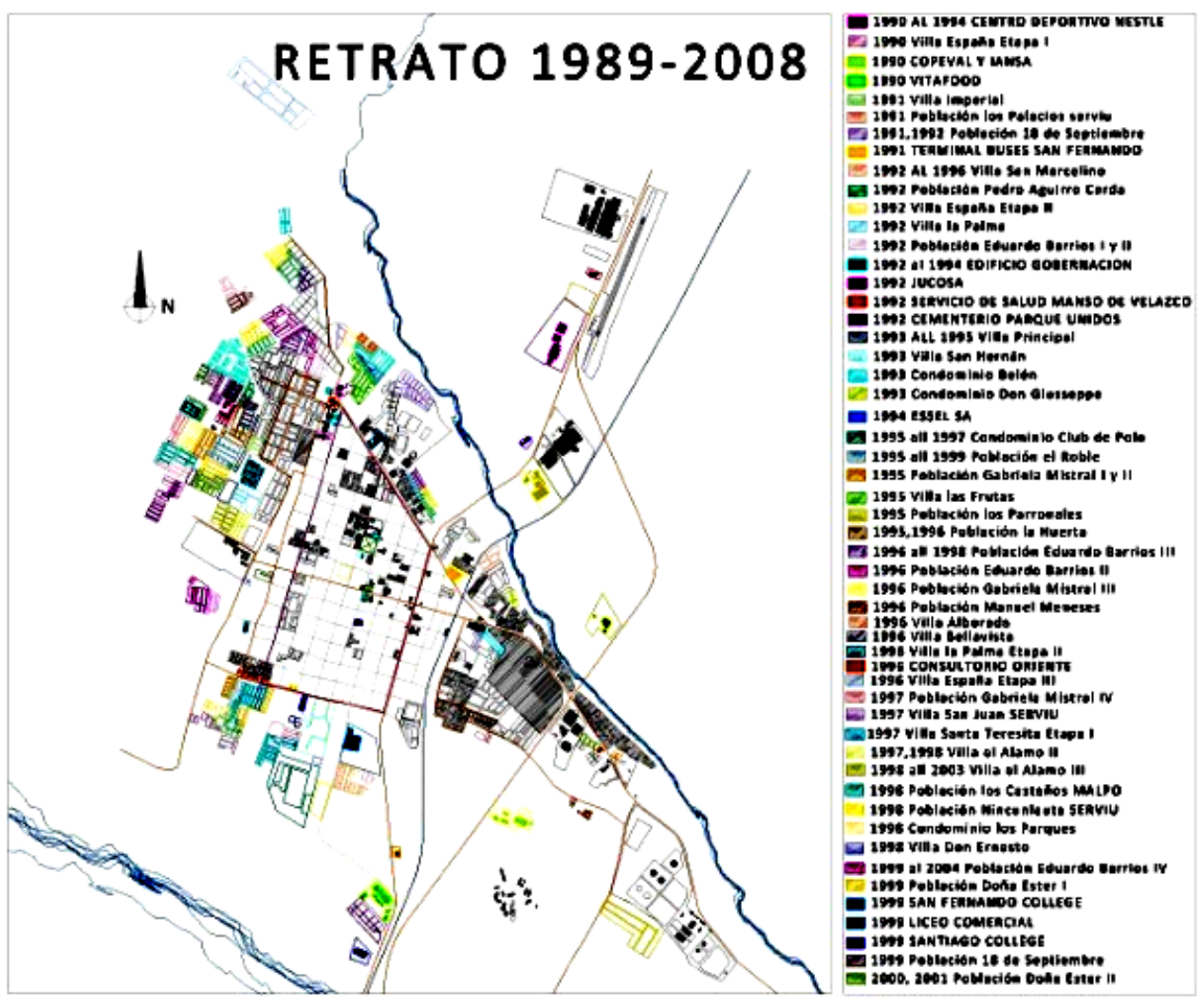

Fig. 8. Retrato de la ciudad período 1989-2008. Fuente: elaboración M. Saavedra.

En los costados del Condominio Club de Polo se emplazan conjuntos cerrados y de carácter privado dotados de espacios comunes a pequeña y gran escala, estableciendo una nueva zona habitacional con cualidad de parques y viviendas de buena factura.

A mediados de 2008 se distinguen 3 áreas:

\section{- Área A. Zona norte de la ciudad}

De trama variada y disposición diversa, la superficie ocupada ya se equipara a la ciudad histórica, el crecimiento define límites que rápidamente se convierten en tramas interiores, por medio de conjuntos residenciales de sucesivas e innumerables etapas.

- Área B. Zona Sur.

Compuesta por unidades de carácter privado y volcadas hacia su interior, determinando espacios vacios entre sí, la ciudad se acerca rápidamente hacia el borde del río Tinguiririca, mostrando una tendencia de crecimiento dispersa. 
- Área C. Consolidación del eje transversal Carretera Panamericana.

La ciudad traspasa los límites del estero Antivero hacia el norte por medio de la actividad agroindustrial, la cual establece nuevas zonas de recorridos a los centros laborales ubicados en la zona de expansión industrial propuesta por el Plan Regulador Comunal de 1998.

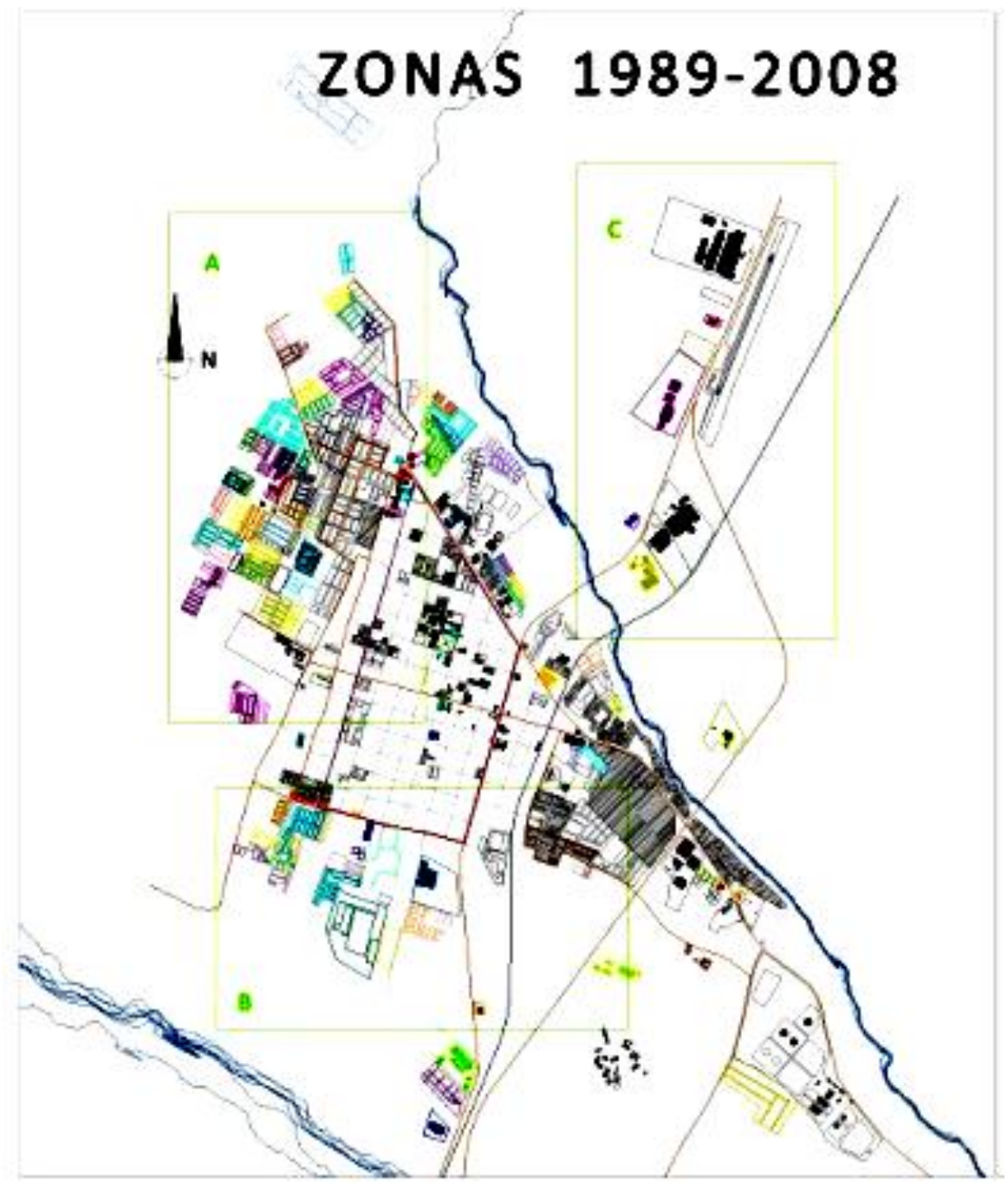

Fig. 9. Tres zonas o áreas de crecimiento 1989-2008. Fuente: elaboración M. Saavedra.

La ciudad sufre las consecuencias del crecimiento explosivo que se produce primero en paños-isla, los cuales se acercan paulatinamente hacia los bordes de la ciudad y en etapas sucesivas hasta rellenar los espacios vacios. 
Aparecen conjuntos de alta densidad y monótona repetición de volúmenes, los cuales se emplazan a modo de salientes y entrantes, originando un tejido urbano formado por unidades que se agrupan de formas diversas, reconociendo en primer lugar sus estructuras viales y luego sus partes desconectadas, importadas de modelos urbanos ajenos.

San Fernando construye viviendas pero no el entorno que las contiene, se transforma en una ciudad de fragmentos.

\section{Conclusión.}

En el período 1942-1972 la ciudad evoluciona hacia una organización vial. Por otra parte, la calidad de vida de los Sanfernandinos ${ }^{2}$ se ve mejorada gracias a la progresiva construcción de equipamientos. La ciudad comienza a traspasar sus límites fundacionales y avanza hacia el estero Antivero por medio de tejidos caprichosos y expuestos a los riesgos naturales (crecidas invernales): la ciudad histórica y su borde estero.

En el periodo 1973-1989 la planificación se ve relegada a una pausa obligada y San Fernando se desarrolla en base a las reglas del mercado, liberando el límite urbano. La ciudad crece entre los cursos de agua Antivero y Tinguiririca y se proyecta hacia el norte en las áreas adyacentes a la Panamericana Sur; conformando una agrupación de importancia e intercambio económico intercomunal: la ciudad de traspaso.

En el periodo 1990-2008, San Fernando va completando progresivamente el área del borde del mencionado estero. El crecimiento de la ciudad está condicionado por sus cursos de agua y determinada por la implantación de industria peligrosa hacia la Cordillera. Esto ha provocado un crecimiento hacia el noroeste de la ciudad ocupando terrenos de excelente calidad agrícola y por ende de gran capacidad de absorción de agua en épocas estivales, asentando la ciudad precisamente en zonas inundables y ocupando los mejores suelos de cultivo al incluirlos dentro de los límites urbanos.

En sus contornos, San Fernando se fortalece y continúa su crecimiento en base a unidades que se adosan, carentes de relaciones espaciales comunes y en órdenes repetitivos, desplazando sus límites a corto plazo, integrando rápidamente la periferia a la trama interna: la ciudad de fragmentos.

La ciudad crece en base a hiladas casa -calle-casa, las cuales en siguientes etapas se unen por sus patios.

San Fernando se resiste a densificar su centro con edificaciones en altura, lo que traería consigo una mejor calidad de vida dada por la cercanía a sus equipamientos y centros comerciales, además de un incremento en la plusvalía de los suelos urbanos.

\footnotetext{
${ }^{2}$ Gentilicio de los habitantes de San Fernando. 
El uso de suelo casi exclusivo de viviendas en la periferia segrega el espacio urbano, estableciendo distintas categorías de San Fernandinos según la facilidad o dificultad de acceder a la zona de trabajo, de atención de salud, de educación, etc.

A pesar de la expansión urbana que ha protagonizado la ciudad, se ha perdido la oportunidad de generar nuevas propuestas de espacios públicos: ya sean parques, plazas o avenidas arboladas. Es por esto que para los habitantes se mantienen siempre presentes los antiguos trazados urbanos como referencia; ya que en los nuevos tejidos -de órdenes arbitrarios- no existen aperturas visuales y tampoco proyecciones de espacios que relacionen unas unidades con otras. El casco histórico y sus límites siguen teniendo una jerarquía urbana que no tienen las zonas nuevas de expansión y crecimiento.

Es evidente que el pasado de la ciudad, no logra proyectarse fuera de su núcleo de implantación, ya que los habitantes modernos, han proyectado la ciudad con recortes de otros lugares, ignorando la potencialidad de esta ciudad de historia siempre presente de manera silenciosa y cotidiana en sus grandes avenidas.

San Fernando define básicamente tres etapas de desarrollo a lo largo de su historia: la primera de concentración urbana determinada por su casco histórico; en segundo lugar la descentramiento por medio del crecimiento de sus contornos principalmente hacia el borde estero Antivero; y finalmente se define una tercera etapa de dispersión de la silueta urbana perdiendo definición y control de sus perímetros.

La ciudad en el presente es reconocible por medio de sus partes, ya que es un todo funcional, pero no un todo relacionado. Sus fragmentos urbanos se suceden, se separan y se disgregan al recorrerla en una suerte de mosaico urbano; que a gran escala se caracteriza por la discontinuidad de la forma urbis, esto es la dispersión de fragmentos sobre el territorio.

La discontinuidad morfológica y deslocalización de estos fragmentos o crecimientos recientes "no solo implica ausencia de continuidad, sino también, de contigüidad, es decir, de cercanía con el origen" (Salinas, 2010:51).

El trabajo de investigación ha buscado recorrer los distintos momentos y secuencias a lo largo de la evolución reciente de la ciudad, pretendiendo proveer un esbozo acerca de cómo ésta se ha desarrollado por medio de un registro detallado de la historia contemporánea de la ciudad. El conocimiento de su construcción material es al mismo tiempo cultural, puede aportar a la reinterpretación de la identidad y a la elaboración de los proyectos de futuro en los que también se hace presente la historia.

\section{Bibliografía.}

BODINI, Hugo. Geografía Urbana. 1a ed, Santiago: Instituto Geográfico Militar Tomo X Colección de Geografía de Chile, 1985. 227 p. ISBN: 988896.

DE RAMÓN, Armando. Santiago de Chile (1541-1991): historia de una sociedad urbana. Santiago de Chile: Editorial Sudamericana, 2000. 287 p. ISBN 956-262-118-9. 
San Fernando en el siglo XX: de la ciudad concentrada al mosaico urbano The city of San Fernando in the 20th Century: from a compact city to an urban mosaic

http://revistaurbanismo.uchile.cl

Disponible en http://www.memoriachilena.cl/archivos2/pdfs/MC0007069.pdf [Consulta: 16 de septiembre de 2010]

DIRECCIÓN DE OBRAS MUNICIPALES (DOM) SAN FERNANDO. Carpetas de Expedientes de construcción. I. Municipalidad de San Fernando.

FERNÁNDEZ, José. "Metodología etnográfica de la historia urbana", en: Revista de Antropología Social, Madrid, editada por Servicio de Publicaciones Universidad Complutense de Madrid, 2001, $\mathrm{n}^{\circ}$ 10, pp. 17-28. ISSN: 1131-555 X Disponible en: http://www.ucm.es/BUCM/revistas/cps/1131558x/articulos/RAS00101110017A.PDF [Consulta: 18 de septiembre de 2008]

GARCÍA, Aurora. "La Ciudad Objeto de Estudio Pluridisciplinar", p. 11-33. En: GARCÍA, Aurora. (Ed.) Prácticas de Geografía Urbana 1: La Ciudad Objeto de Estudio Pluridisciplinar. Barcelona: Oikos-Tau, 1995. 177 p. ISBN 9788428108591.

GONZÁLEZ Carmen; ROMERO, Hugo. "Desarrollo histórico de la ciudad de San Fernando VI región, Chile", en: Mapping Revista internacional de Ciencias de la Tierra, Madrid, editada por Revista Mapping, S.L., $n^{\circ}$ 100, marzo-abril 2005 (s/p). Disponible en http://www.mappinginteractivo.com/plantilla-ante.asp?id articulo=880 [10 agosto de 2008]. ISSN: 1.131-9.100

GONZÁLEZ Carmen; ROMERO, Hugo y Julia GONZÁLEZ. "Evaluación espacial de la sustentabilidad ambiental en ciudades intermedias. Estudio de caso de la ciudad de San Fernando VI región, Chile", en: Mapping. Revista internacional de Ciencias de la Tierra, Madrid, editada por Revista Mapping, S.L., n 96, Sept.-oct. 2004 (s/p). Disponible en http://www.mappinginteractivo.com/plantilla-ante.asp?id articulo=746 [16 de agosto de 2008]. ISSN: 1.131-9.100

HIDALGO, Rodrigo. La vivienda social en Chile y la construcción del espacio urbano en el Santiago del siglo XX. Santiago: Instituto de Geografía, P. Universidad Católica/Centro de Investigaciones Diego Barrios Arana, 2005. 491 p. ISBN 956-244169-5

INSTITUTO GEOGRÁFICO MILITAR (IGM). Fotografía Aérea de San Fernando, 22 de Enero de 1955.

KORN, Arthur. La historia construye la ciudad (trad. de Norberto A. Chiesa). Buenos Aires: Eudeba, 1963. 234 p.

LORENZO, Santiago. Fuentes para la historia urbana en el Reino de Chile, tomo I. Santiago: Academia Chilena de la Historia (Santiago: Universitaria), 1995. 334 p.

MINISTERIO DE VIVIENDA Y URBANISMO. CHILE. Estudio preinversional de San Fernando, Provincia de Colchagua [libro]. Santiago: [s.n.] Dirección de Planificación del Desarrollo, Oficina Interdisciplinaria para el Desarrollo OIDES, 1969. 58 p. 
http://revistaurbanismo.vchile.cl

MUNIZAGA, Gustavo, Macroarquitectura: Tipologías y Estrategias de Desarrollo Urbano. ( $2^{\mathrm{a}}$ ed.) Santiago: Textos Universitarios, Facultad de Arquitectura, Ediciones Universidad Católica de Chile, 1999. 392 p. ISBN: 9789561405127

OLAVE, Dídima. "El sistema urbano de Chile central. Desafíos sociales y medio ambientales", en: Scripta Nova. Revista electrónica de geografía y ciencias sociales, Barcelona, editada por Universitat de Barcelona, agosto de 2005, vol. IX, núm. 194 (69). http://www.ub.es/geocrit/sn/sn-194-69.htm [ISSN: 1138-9788]

SALINAS, Edison. "Fragmentación urbana y su relevancia en la planificación urbana y territorial actual", en: revista Discusiones Públicas, Santiago, editada por Ignire-Centro de Estudio de Política Pública, 2010, vol. 1, n 1, p. 49-58. ISSN 0718-8099. Disponible en http://www.ignire.cl/articulos/ [Consulta: 18 de septiembre de 2010]

SERVICIO AEROFOTOGRAMÉTRICO (SAF) FUERZA AÉREA DE CHILE. Fotografía aérea de San Fernando, 1978.

VILLAVICENCIO, Judith. El Desarrollo Histórico de la Red Urbana en América Latina: el Caso de Chile. Santiago: P. Universidad Católica de Chile, Centro Interdisciplinario de Desarrollo Urbano y Regional [s.n.], 1971. 23 p.

http://www.sinia.cl/1292/printer-26197.html Política Ambiental de la Región del Libertador Bernardo O'Higgins. [Consulta: 30 de marzo de 2008] 